\title{
Delirium in the elderly
}

\section{A systematic review of pharmacological and non-pharmacological treatments}

\author{
Cecília Carboni Tardelli Cerveira', Cláudia Cristina Pupo², \\ Sigrid De Sousa dos Santos ${ }^{3}$, José Eduardo Mourão Santos ${ }^{3}$
}

\begin{abstract}
Delirium is a common disorder associated with poor prognosis, especially in the elderly. The impact of different treatment approaches for delirium on morbimortality and long-term welfare is not completely understood. Objective: To determine the efficacy of pharmacological and non-pharmacological treatments in elderly patients with delirium. Methods: This systematic review compared pharmacological and non-pharmacological treatments in patients over 60 years old with delirium. Databases used were: MEDLINE (PubMed), EMBASE, Cochrane CENTRAL and LILACS from inception to January $6^{\text {th }}, 2016$. Results: A total of ten articles were selected. The six non-pharmacological intervention studies showed no impact on duration of delirium, mortality or institutionalization, but a decrease in severity of delirium and improvement in medium-term cognitive function were observed. The most commonly used interventions were temporal-spatial orientation, orientation to self and others, early mobilization and sleep hygiene. The four studies with pharmacological interventions found that rivastigmine reduced the duration of delirium, improved cognitive function and reduced caregiver burden; olanzapine and haloperidol decreased the severity of delirium; droperidol reduced length of hospitalization and improved delirium remission rate. Conclusion: Although the pharmacological approach has been used in the treatment of delirium among elderly, there have been few studies assessing its efficacy, involving a small number of patients. However, the improvements in delirium duration and severity suggest these drugs are effective in treating the condition. Once delirium has developed, non-pharmacological treatment seems less effective in controlling symptoms, and there is a lack of studies describing different non-pharmacological interventions.
\end{abstract}

Key words: delirium, elderly, pharmacological, non-pharmacological, systematic review.

\section{DELIRIUM EM IDOSOS: REVISÃO SISTEMÁTICA DE TRATAMENTOS FARMACOLÓGICOS E NÃO-FARMACOLÓGICOS}

RESUMO. Delirium é uma condição comum associada com prognóstico pobre, especialmente em idosos. 0 impacto das diferentes abordagens de tratamento na morbi-mortalidade e bem-estar de longo prazo não é completamente compreendido. Objetivo: Determinar a eficácia dos tratamentos farmacológico e não-farmacológico em pacientes idosos com delirium. Métodos: Esta revisão sistemática comparou tratamentos farmacológicos e não-farmacológicos em pacientes com idade superior a 60 anos com delirium. As bases de dados usadas foram: MEDLINE (PubMed), EMBASE, Cochrane Central e LILACS do início até 6 de janeiro de 2016. Resultados: Dez artigos foram selecionados. Seis estudos com intervenções não farmacológicas não mostraram impacto na duração, mortalidade ou institucionalização, mas houve redução na gravidade do delirium e memlhora na função cognitive de médio prazo. As intervenções mais comumente utilizadas foram orientação temporo-espacial, orientação para si e outros, mobilização precoce e higiene do sono. Os quarto estudos com intervenções farmacológicos acharam que a rivastigmina reduziu a duração, melhorou a função cognitive e reduziu a sobrecarga do cuidador, olanzapine e haloperidol diminuíram a gravidade de delirium e o droperidol diminuiu a duração da hospitalização e na taxa de remissão. Conclusão: Embora a abordagem farmacológica tem sido usada no tratamento de delirium em idosos, há estudos que avaliam sua eficácia com limitado número de pacientes. Todavia, a melhora na duração e gravidade sugerem que estas drogas são efetivas no tratamento desta condição. Uma vez que o delirium esteja instalado, o tratamento não farmacológico parece ser menos efetivo no controle dos sintomas, há uma falta de estudos que discriminem as diferentes intervenções não-farmacológicas.

Palavras-chave: delirium, idosos, farmacológico, não-farmacológico, revisão sistemática.

This study was conducted for a Integrated Master's Degree in Medicine at the Abel Salazar Institute of Biomedical Sciences (ICBAS), University of Porto, Porto, Portugal. ${ }^{1}$ MD, Unidade Básica de Assistência à Saúde, Universidade de São Paulo - Campus de São Carlos, SP, Brazil. MD, Departamento de Medicina, Centro de Ciências Biológicas e da Saúde, Universidade Federal de São Carlos, SP, Brazil. MD, PhD, Departamento de Medicina, Centro de Ciências Biológicas e da Saúde, Universidade Federal de São Carlos, SP, Brazil.

Cecília Carboni Tardelli Cerveira. Avenida Miguel Damha, 1400 / casa 329 - Mail box: 13565-251. E-mail: cetardelli@hotmail.com

Disclosure: The authors report no conflicts of interest.

Received June 22, 2017. Accepted in final form August 04, 2017. 


\section{INTRODUCTION}

Delirium is an acute confusional state characterized by consciousness disorder, decline in cognitive function and attention, abrupt onset and fluctuating course. ${ }^{1}$ The condition is associated with a rapid reduction in brain function and is usually triggered by diseases with systemic involvement. It has a considerable impact on morbidity and lethality, especially among the elderly..$^{-4}$

The incidence of delirium is $1-2 \%$ in the general population, $22 \%$ in hospitalized patients, $11-62 \%$ in postoperative patients (depending on type of surgery and study population) and up to $80 \%$ among patients in intensive care units. ${ }^{2,57}$ In the elderly, the incidence reaches $50 \%$ in hospitalized patients. ${ }^{6,7}$ Nevertheless, many cases go undiagnosed.

Delirium is associated with many negative outcomes including increased length of hospital stay and risk of complications; higher mortality ( $8 \%$ versus $1 \%$ ), institutionalization rates ( $16 \%$ versus $3 \%)$, and risk of permanent cognitive decline. ${ }^{4-10}$ In intensive care units, the condition is associated with an increased length of stay and longer duration of mechanical ventilation. ${ }^{11}$

The presence of delirium accelerates the ageing of the brain, elevates the risk of dementia in predisposed individuals and can mask prior cognitive impairment not yet diagnosed. . $^{8,9,12}$

The major risk factors for delirium are previous cognitive impairment, history of alcohol abuse and advanced age ( $>70$ years). ${ }^{6,13}$ The presence of dementia is the major risk factor of delirium in the elderly. Polypharmacy (use of five or more medications) is the commonest independent and reversible risk factor of delirium. Sedatives, analgesics and anticholinergic drugs are the most commonly involved. Other risk factors involved are: severity of the underlying disease, infections, fractures at admission and physical restriction. The duration of delirium in the elderly is generally longer and the symptoms more severe. ${ }^{2,6}$

The non-pharmacological approach has been the most used strategy for preventing delirium. ${ }^{6}$ A nonpharmacological intervention package known as the Hospital Elder Life Program - HELP (cognitive impairment management, sleep hygiene, early mobility, visual and hearing support, hydration) has demonstrated effects on the incidence and total days with delirium. However, once delirium has developed, HELP seems to have no impact on severity and recurrence. ${ }^{13,14}$

Studies using different pharmacological approaches for delirium prevention (haloperidol, olanzapine, risperidone, rivastigmine, ketamine, dexmedetomidine, morphine, donepezil and midazolam) have not found sufficient evidence of effectiveness. ${ }^{6}$ However, melatonin and its agonist ramelteon have been found to be potentially beneficial in the prevention of delirium in the elderly. ${ }^{5}$

Treatment of delirium is mainly based on resolution of the underlying condition combined with non-pharmacological interventions and specific pharmaceutical interventions..$^{15}$ Consequently, delirium diagnosis and early identification of its causal and predisposing factors depend on healthcare team training. After discharge, patients who have developed delirium require monitoring given the high possibility of a further episode. ${ }^{2}$ To date, no specific treatment for delirium has been approved by the Food and Drug Administration. . $^{16,17}$

For patients in psychic distress or too restless, at risk of harming themselves, caregivers or health professionals, antipsychotics have been recommended based on clinical evidence and expert opinion. ${ }^{2}$ Haloperidol, a typical antipsychotic and neuroleptic, has been the most studied and routinely used medication. It blocks cortical and nigrostriatal dopamine receptors (D2 antagonist), and disinhibits acetylcholine. It should be initiated at the smallest possible dose for the shortest possible period. Side effects include extrapyramidal symptoms, akathisia, neuroleptic malignant syndrome, tardive dyskinesia, glucose and cholesterol changes, cardiac arrhythmias, and venous thromboembolism. ${ }^{17}$

In a systematic review of studies comparing atypical antipsychotics (amisulpride, quetiapine, olanzapine and risperidone) with typical antipsychotics (haloperidol) for the treatment of delirium in a wide range of clinical conditions, all medications have been proven effective and safe with no significant difference between agents. ${ }^{1}$

Perioperative melatonin has been compared to no drug (control), midazolam or clonidine in prevention and treatment of postoperative delirium. Patients receiving melatonin had a lower incidence of postoperative delirium. In addition, postoperative delirium was successfully treated with melatonin in $58 \%$ of patients. However, this response was not controlled, and it is difficult to impute causality. ${ }^{18,19}$

There is no evidence to support the use of benzodiazepines for the treatment of cases of delirium not related to alcohol withdrawal. These agents may have a deleterious effect, triggering delirium, increasing the risk of falls, as well as causing changes in memory and withdrawal symptoms. 2,15

Non-pharmacological interventions, characterized by a multi-professional activity, have been the main 
approach used in patients with delirium. Although there are many therapeutic approaches for delirium (cognitive impairment management, sleep hygiene, early mobility, visual and hearing support, hydration, environment interventions, orientation interventions, familiarity interventions, communication strategies, pain control etc.); the importance, impact, and specificity of each of these interventions on morbidity, mortality and quality of life after discharge is unclear. ${ }^{15-17}$

The main goal of the present systematic review was to identify studies investigating the effectiveness of pharmacological and non-pharmacological interventions on the duration of delirium in patients aged 60 years or older. As secondary goals, the effects of these interventions on length of hospital stay, lethality, incidence of complications and functional decline were evaluated.

\section{METHODS}

Our study was designed according to the recommendations of Higgins \& Green (2011) "Cochrane handbook for systematic reviews of interventions". ${ }^{20}$ The databases used were MEDLINE (PubMed), EMBASE, Cochrane Library (CENTRAL) and LILACS. All databases were searched from inception to January $6^{\text {th }}, 2016$. There was no restriction on language. The following major Medical Subject Headings terms were included: ("delirium" OR "acute confusion" OR "confusion state" OR "confusional state" OR "acute confusion state" OR "acute confusional state") AND ("elderly" OR "aged" OR "ancient" OR "geriatric") AND ("treatment" OR "therapy"). The filters "Clinical Trial" OR "Trial" were used for MEDLINE; "Trial" for the Cochrane Library; and "controlled clinical trial" OR "randomized controlled trial" for EMBASE. No filter was used for LILACS.

Eligible studies for inclusion were randomized or controlled clinical trials in which patients were 60 years or older with the diagnosis of delirium based on the Diagnostic and Statistical Manual of Mental Disorders (DSM), International Classification of Diseases-10 (ICD10) or the use of a validated instrument for the diagnosis of delirium.

The primary outcome was the duration of delirium. The secondary outcomes were: length of hospital stay, need of physical restraint, lethality, functional decline and the incidence of complications.

After listing articles retrieved by the databases, all references were exported to the EndNote Web library. Two authors (CCTC and CCP) were then responsible for reading the titles in parallel using the EndNote Web program. Duplicates were excluded and titles and abstracts of articles were subsequently selected according to the established criteria. If the abstract was not available but the title was suggestive of inclusion, the article remained in the base for the next step of assessing eligibility by reading the full-text. The same held true in cases of doubt or disagreement among reviewers. The reason for the exclusion of each abstract and full article was recorded for the composition of the article selection algorithm.

The decision to exclude studies was reached through consensus and based on the established criteria, including participants, interventions, comparisons, outcomes, and study design (PICOS). A third reviewer (SSS) was consulted in case of doubt or disagreement between the reviewers.

Acceptable studies had a clear description of design, age range, diagnosis, intervention and outcome. The review process excluded studies with unclear designs, improper control group, younger or adult-only samples, insufficient outcome data, unclear diagnosis of delirium at study admission, or insufficient outcome data.

As required, non-English language articles were translated into English using an online translation service (Google Translator), with subsequent revision by a native speaker (Russian, Chinese and Korean).

The data from the selected articles were extracted using a standard form, including identification, study design, characteristics of the study population, type of intervention, outcomes, main results and comments. Study authors were contacted for additional data when needed.

\section{RESULTS}

A total of 2154 articles were selected for review, comprising 431 studies in PubMed; 1170 studies in EMBASE; 481 studies in the Cochrane Library (CENTRAL); and 72 studies in LILACS. We selected 10 studies evaluating 1588 patients. The selection algorithm is depicted in Figure 1.

The selected studies are described in Table 1 , according to study design, clinical and epidemiological characteristics of the study population, prior prevalence of dementia, delirium diagnostic criteria, interventions, observed outcomes and results.

A meta-analysis could not be performed due to several factors, including the variability in interventions proposed as non-pharmacological treatments, in doses and types of drugs used as pharmacological treatment, intervention strategies (individual or specific ward), parameters and time interval used for outcome evaluation, and type of comparison group employed. 


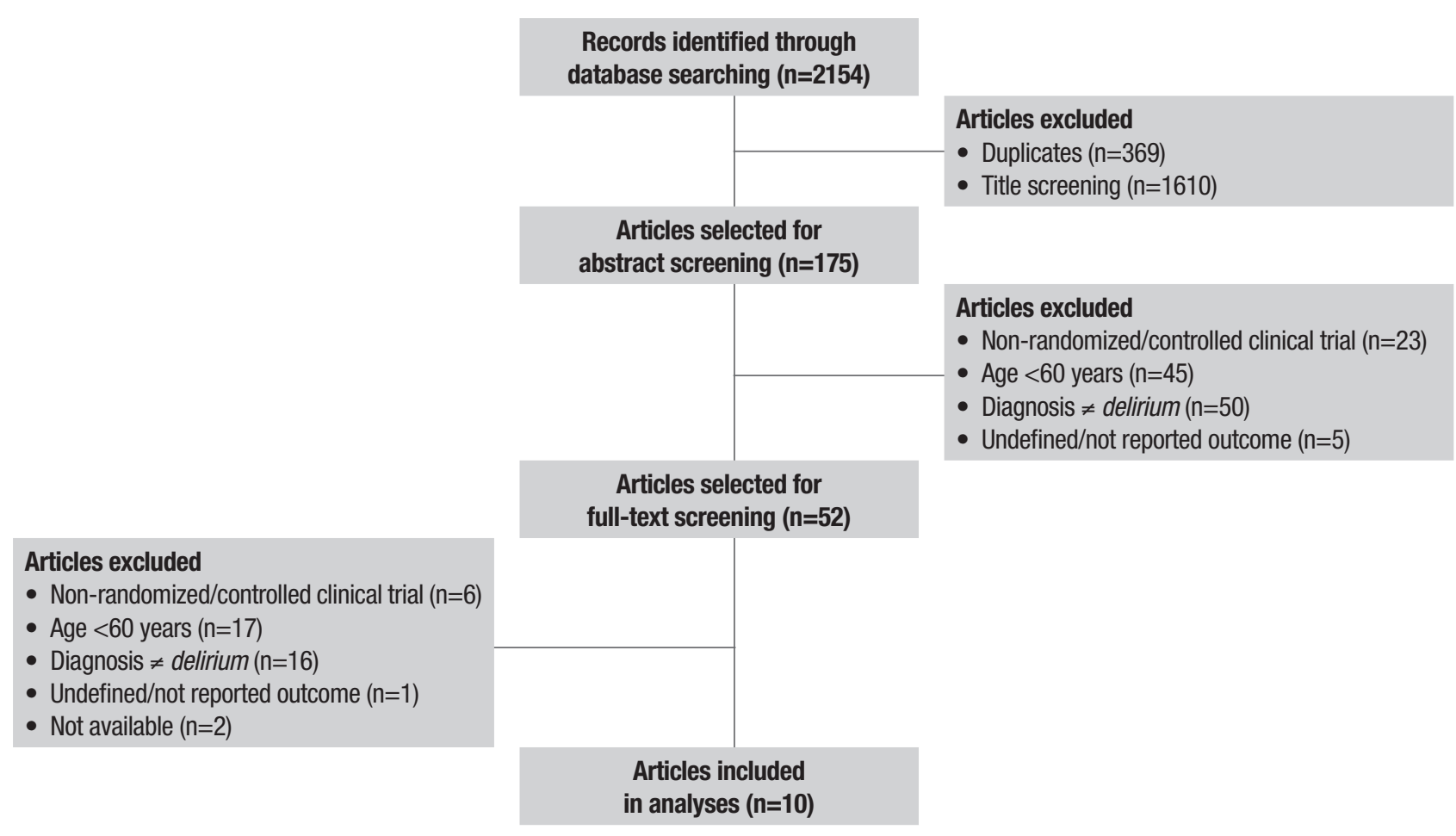

Figure 1. PRISMA flow diagram of study inclusion and exclusion.

\section{DISCUSSION}

Both pharmacological and non-pharmacological interventions have shown impact on health status of elderly patients with delirium. Non-pharmacological interventions, while not significantly affecting the duration and severity of delirium, or mortality; did contribute to functional improvement in the medium term and reduced complications. Several medications were studied to control agitation of hyperactive delirium, with demonstrable improvement in delirium severity, duration and remission rate, positively affecting length of hospital stay and caregiver burden.

Interventions that combine cognitive impairment management, sleep hygiene, early mobility, visual and hearing support, and hydration care (HELP protocol) with light therapy have contributed to significant improvement in functional status and to absence of physical restraints for the care of delirium in geriatric wards. $^{21}$

Strategy combining orientation interventions (clock, calendar, day's schedule chart, visual and hearing support, language interpreters); familiarity interventions with objects and family members; physiotherapy; hip protectors, and nutritional supplements with the prescription of cholinesterase inhibitors as needed and restriction of the use of neuroleptics provided more pro- nounced and rapid reduction in the severity of delirium and cognitive improvement after discharge. ${ }^{30}$

Intervention combining entertainment (radio, television); sleep hygiene (light and sound); orientation interventions; familiarity interventions with the presence of objects and people they know; attention to visual and auditory acuity, communication intervention with visual contact, empathy, calm speech; early mobility stimulation, and psychiatric and geriatric specialist consultation was associated with more rapid cognitive improvement. ${ }^{29}$

The reviewed studies have a small number of participants, a possible underdiagnosis of delirium and an inclusion of frail populations. The difference in dementia prevalence among the groups might also have influenced the outcome, since it is a risk factor and affects delirium prognosis.

Non-pharmacological interventions for delirium were generally applied together, encompassing several domains, and seem less effective in controlling symptoms. It was difficult to discriminate the effectiveness of each individual therapeutic approach.

Pharmacological intervention studies have generally included cholinesterase inhibitors, typical (haloperidol and droperidol) and atypical antipsychotics (olanzapine and others). Despite the fact that there have been few 


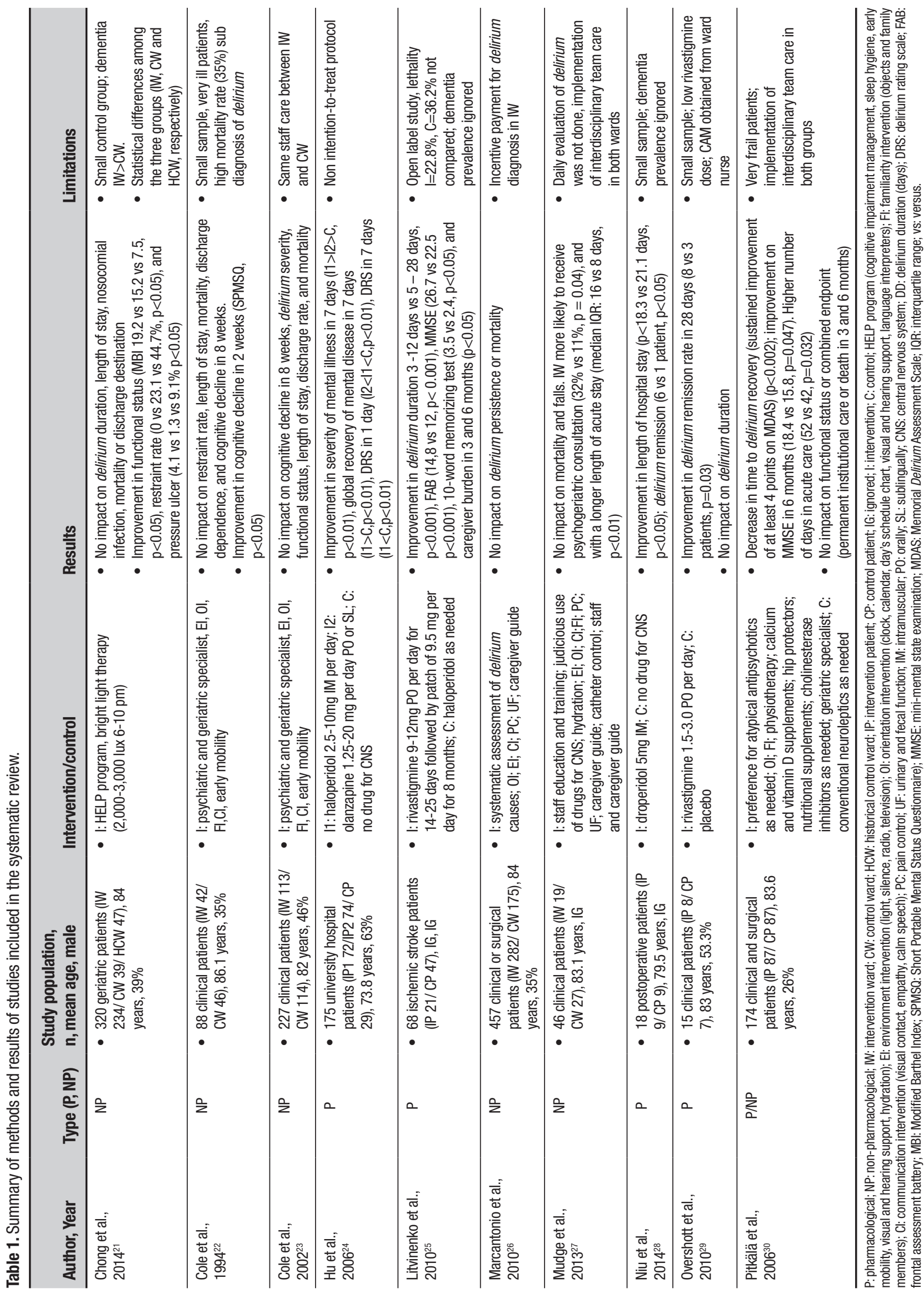


published studies, involving a small number of cases, pharmacological treatment significantly improved delirium duration and severity.

Rivastigmine, a cholinesterase inhibitor used for treatment of dementia, decreased the duration of delirium, improved cognitive function and reduced caregiver stress in older patients with ischemic stroke. ${ }^{25}$ Both olanzapine and haloperidol decreased the severity of delirium in elderly patients admitted to medical wards, and recovery was slightly faster with olanzapine. ${ }^{24}$ The use of droperidol reduced the length of hospital stay and increased postoperative delirium remission rate in elderly patients with colorectal cancer. ${ }^{28}$

Once old patients have developed acute cognitive decline, delirium must be diagnosed and promptly treated. The therapy includes detecting and treating underlying critical conditions, providing pharmacological control of agitation preferentially with atypical antipsychotics, improving cognitive function with cholinesterase inhibitors, and associating multiple interven- tions to hydrate, restore the sleep-wake cycle, provide early mobility, support visual and hearing deficits, provide temporal orientation and familiarity sensation, and improve communication. Therapeutic action in multiple domains can decrease severity of mental illness, time to delirium recovery, and guarantee better long-term cognitive function.

Author contributions. CCTC and SDSS conceived the study and participated in its design; CCTC and CCP performed the review of the articles; SDSS and JEMS developed the search strategies and supported the review process; CCTC, CCP and SDSS drafted the manuscript. All authors read and approved the final version of the manuscript.

Acknowledgments. We would like to thank the Serviço de Biblioteca e Informação "Prof. Bernhard Gross", Instituto de Física de São Carlos, Universidade de São Paulo, for the database search support and supply of articles.

\section{REFERENCES}

1. Wang HR, Woo YS, Bahk WM. Atypical antipsychotics in the treatment of delirium. Psychiatry Clin Neurosci 2013;67(5):323-31.

2. van Munster BC, de Rooji S. Delirium:a synthesis of current knowledge. Clin Med 2014;14(2):192-5.

3. Martinez F, Tobar C, Hill N. Preventing delirium:should non-pharmacological, multicomponent interventions be used? A systematic review and meta-analysis of the literature. Age Ageing 2015;44(2):196-204

4. Androsova G, Krause R, Winterer G, Schneider R. Biomarkers of postoperative delirium and cognitive dysfunction. Front Aging Neurosci 2015;7:112.

5. Chakraborti D, Tampi DJ, Tampi RR. Melatonin and Melatonin Agonist for Delirium in the Elderly Patients. Am J Alzheimers Dis Other Demen 2015:30 (2):119-29.

6. Inouye SK, Westendorp RG, Saczynski JS. Delirium in elderly people. Lancet 2014;383(9920):911-22.

7. Maldonado JR. Neuropathogenesis of delirium:review of current etiologic theories and common pathways. Am J Geriatr Psychiatry 2013; 21(12):1190-222.

8. Witlox J, Eurelings LSM, de Jonghe JFM, Kalisvaart KJ, Eikelenboom P, van Gool WA. Delirium in Elderly Patients and the risk of Postdischarge Mortality, Institutionalization, and Dementia: a Meta-analysis. JAMA 2010; 304(4):443-51

9. Davis DH, Muniz Terrera G, Keage H, Rahkonen T, Oinas M, Matthews $\mathrm{FE}$, et al. Delirium is a strong risk factor for dementia in the oldest-old:a population-based cohort study. Brain 2012;135(Pt 9):2809-16.

10. Girard TD, Jackson JC, Pandharipande PP, Pun BT, Thompson JL, Shintani AK, et al. Delirium as a predictor of long-term cognitive impairment in survivors of critical illness. Crit Care Med 2010;38 (7):1513-20.

11. Salluh JI, Wang H, Schneider EB, Nagaraja N, Yenokyan G, Damluji A, et al. Outcome of delirium in critically ill patients:systematic review and metaanalysis. BMJ 2015;350:h2538.

12. Pandharipande PP, Girard TD, Jackson JC, Morandi A, Thompson JL, Pun BT, et al. Long-term cognitive impairment after critical illness. N Engl J Med 2013;369(14):1306-16.

13. Inouye SK, Bogardus ST, Baker DI, Leo-Summers L, Cooney LM. The Hospital Elder Life Program:a model of care to prevent cognitive and functional decline in older hospitalized patients. Hospital Elder Life Program. J Am Geriatr Soc 2000;48(12):1697-706.

14. Inouye SK, Bogardus Jr ST, Charpentier PA, Leo-Summers L, Acampora $D$, Holford TR, et al. A multicomponent intervention to prevent delirium in hospitalized older patients. N Engl J Med 1999;340(9):669-76.

15. Javedan $\mathrm{H}$, Tulebaev S. Management of common postoperative complications: delirium. Clin Geriatr Med 2014;30(2):271-8.

16. Irwin SA, Pirrello RD, Hirst JM, Buckholz GT, Ferris FD. Clarifying delirium

management:practical, evidenced-based, expert recommendations for clinical practice. J Palliat Med 2013;16(4):423-35.

17. Hipp DM, Ely EW. Pharmacological and nonpharmacological management of delirium in critically ill patients. Neurotherapeutics 2012:9(1): 158-75.

18. Sultan SS. Assessment of role of perioperative melatonin in prevention and treatment of postoperative delirium after hip arthroplasty under spinal anesthesia in the elderly. Saudi J Anaesth 2010;4:169-73.

19. Inouye SK, Robinson T, Blaum C, Busby-Whitehead J, Boustani M, Chalian A, et al. Postoperative delirium in older adults: best practice statement from the American Geriatrics Society. J Am Coll Surg 2015; 220(2):136-48.e1.

20. Higgins JPT, Green S. Cochrane Handbook for Systematic Reviews of Interventions Version 5.1.0 [updated March 2011]. The Cochrane Collaboration, 2011. Available from http://handbook.cochrane.org.

21. Chong MS, Chan M, Tay L, Ding YY. Outcomes of an innovative model of acute delirium care:the Geriatric Monitoring Unit (GMU). Clin Interv Aging 2014;9:603-12

22. Cole MG, Primeau FJ, Bailey RF, Bonnycastle MJ, Masciarelli F, Engelsman $F$, et al. Systematic intervention for elderly inpatients with delirium: $A$ randomized trial. CMAJ 1994;151(7):965-70.

23. Cole MG, McCusker J, Bellavance F, Primeau FJ, Bailey RF, Bonnycastle $\mathrm{MJ}$, et al. Systematic detection and multidisciplinary care of delirium in older medical inpatients:a randomized trial. CMAJ 2002;167(7):753-9.

24. $\mathrm{Hu} H$, Deng $W$, Yang $H$, Liu $Y$. Olanzapine and haloperidol for senile delirium: A randomized controlled observation. Chinese Journal of Clinical Rehabilitation 2006;10(42):188-90.

25. Litvinenko IV, Odinak MM, Khlystov luV, Perstnev SV, Fedorov BB. Efficacy and safety of rivastigmine (exelon) in the confusion syndrome in the acute phase of ischemic stroke. Zh Nevrol Psikhiatr Im S S Korsakova 2010;110(11 Pt2):36-41.

26. Marcantonio ER, Bergmann MA, Kiely DK, Orav EJ, Jones RN. Randomized trial of a delirium abatement program for postacute skilled nursing facilities. J Am Geriatr Soc 2010;58(6):1019-26.

27. Mudge AM, Maussen C, Duncan J, Denaro CP. Improving quality of delirium care in a general medical service with established interdisciplinary care:a controlled trial. Intern Med J 2013;43(3):270-7.

28. Niu W-B, Li Z-Y, Zhang X-N, Zhang J, Wang G-Y, Yu Y-M. Postoperative delirium in elderly patients with colorectal cancer:Risk factors and treatment. World Chinese Journal of Digestology 2014;22(34):5381-4.

29. Overshott R, Vernon M, Morris J, Burns A. Rivastigmine in the treatment of delirium in older people:a pilot study. Int Psychogeriatr 2010; 22(5):812-8.

30. Pitkala KH, Laurila JV, Strandberg TE, Tilvis RS. Multicomponent geriatric intervention for elderly inpatients with delirium: a randomized, controlled trial. J Gerontol A Biol Sci Med Sci. 2006;61(2):176-81. 